\title{
Characterising the spatial dynamics of sympatric Aedes aegypti and Aedes albopictus populations in the Philippines
}

Jennifer Duncombe ${ }^{1}$, Fe Espino², Kristian Marollano ${ }^{3}$, Aldwin Velazco ${ }^{2}$, Scott A. Ritchie Wenbiao $\mathrm{Hu}^{1}$, Philip Weinstein ${ }^{5}$, Archie C. A. Clements ${ }^{1}$

${ }^{1}$ Infectious Disease Epidemiology Unit, School of Population Health, University of Queensland, Herston, Australia; ${ }^{2}$ Parasitology Department, Research Institute for Tropical Medicine, Muntinlupa City, Metro Manila, Philippines; ${ }^{3}$ Medical Entomology Department, Research Institute for Tropical Medicine, Muntinlupa City, Metro Manila Philippines; ${ }^{4}$ School of Public Health, Tropical Medicine and Rehabilitation Sciences, James Cook University, Cairns, Australia; ${ }^{5}$ Barbara Hardy Institute, University of South Australia, Adelaide, Australia

\begin{abstract}
Entomological surveillance and control are essential to the management of dengue fever (DF). Hence, understanding the spatial and temporal patterns of DF vectors, Aedes (Stegomyia) aegypti (L.) and Ae. (Stegomyia) albopictus (Skuse), is paramount. In the Philippines, resources are limited and entomological surveillance and control are generally commenced during epidemics, when transmission is difficult to control. Recent improvements in spatial epidemiological tools and methods offer opportunities to explore more efficient DF surveillance and control solutions: however, there are few examples in the literature from resource-poor settings. The objectives of this study were to: (i) explore spatial patterns of Aedes populations and (ii) predict areas of high and low vector density to inform DF control in San Jose village, Muntinlupa city, Philippines. Fortnightly, adult female Aedes mosquitoes were collected from 50 double-sticky ovitraps (SOs) located in San Jose village for the period June-November 2011. Spatial clustering analysis was performed to identify high and low density clusters of Ae. aegypti and Ae. albopictus mosquitoes. Spatial autocorrelation was assessed by examination of semivariograms, and ordinary kriging was undertaken to create a smoothed surface of predicted vector density in the study area. Our results show that both Ae. aegypti and Ae. albopictus were present in San Jose village during the study period. However, one Aedes species was dominant in a given geographic area at a time, suggesting differing habitat preferences and interspecies competition between vectors. Density maps provide information to direct entomological control activities and advocate the development of geographically enhanced surveillance and control systems to improve DF management in the Philippines.
\end{abstract}

Keywords: dengue, Aedes, surveillance, control, Philippines.

\section{Introduction}

Dengue fever (DF) is the principal arbovirus in the Philippines. In 2010, reported DF incidence ranged from 36 cases per 100,000 population in the southern Autonomous Region in Muslim Mindanao to 372 cases per 100,000 population in the northern Cordillera Administrative Region (Duncombe et al., 2012). DF is endemic in the Philippines and epidemics occur perennially, generally during the wet season (June-November) when environmental conditions are ideal for the proliferation of the DF vectors Aedes (Stegomyia) aegypti (L.) and Ae. (Stegomyia) albopictus (Skuse).

Corresponding author:

Jennifer Duncombe

Infectious Disease Epidemiology Unit

Level 4, Public Health Building, School of Population Health

The University of Queensland, Herston, QLD 4006 Australia

Tel. +6140406 6753

E-mail: j.duncombe@uq.edu.au
The introduction of Ae. aegypti to Asia in the 19th century led to this species becoming the dominant DF vector in cities, in which it was better adapted than native Ae. albopictus (Lounibos, 2002; Gratz, 2004). Since then, Ae. albopictus has adjusted to urban environments, though it still favours areas of dense vegetation, and the two species reside sympatrically throughout Asia. Although Ae. albopictus is arguably the better larval competitor, Ae. aegypti is considered the more competent DF transmitter, perhaps due to its anthropophilic behaviour, and thus, is acknowledged as the primary DF vector (Gubler and Kuno, 1997).

Evidence for Ae. albopictus being a less efficient vector of DF has led to hypotheses about the public health benefits of Ae. albopictus replacing Ae. aegypti populations throughout the world (Lambrechts et al., 2010). However, Ae. albopictus should not be dismissed as a mere pest: it is a competent viral transmitter and has been shown to be more tolerant of cold conditions than Ae. aegypti; overwintering in areas 
with minimum temperatures below $0{ }^{\circ} \mathrm{C}$ (Lounibos, 2002). Therefore, in the near future we can expect that Ae. albopictus - and ultimately DF - will expand to areas previously uninhabited by vector competent Aedes mosquitoes, and the classic DF transmission season seen in some cooler tropical countries may be extended. As a result, greater morbidity and mortality associated with DF and other viruses transmitted by Ae. albopictus, such as chikungunya, is likely (Powers and Logue, 2007). Further, increasing co-circulation of DF virus serotypes in human populations with specific herd immunity may increase the incidence of dengue hemorrhagic fever (DHF) and dengue shock syndrome (DSS), which are more severe forms of DF resulting from secondary infection with a different serotype (WHO, 2012).

The development of a publicly available vaccine is underway. In the meantime, DF management relies on entomological surveillance and control (Ooi et al., 2006; Morrison et al., 2008). Since the decentralization of the Philippines health system in the 1990s, local authorities have assumed responsibility for health system delivery and financing (Bossert and Beauvais, 2002). Consequently, DF management, including entomological surveillance and control, depends on locallevel support and varies considerably between localities within the country (van den Berg et al., 2012). Because vector control strategies are time-consuming and expensive, they are generally only undertaken during epidemics and may include: physical removal of breeding sites, indoor residual spraying (IRS), outdoor spraying (fogging), and community education.

As geographical information systems (GIS) technology and geostatistical methods progress and become more accessible, knowledge of the spatial dynamics of Aedes populations increases and new opportunities to explore integrated surveillance and control solutions for DF-affected countries are presented (Eisen and Lozano-Fuentes, 2009; Higa, 2011). Commonly, GIS are used for visualising the spread and density of vector breeding sites, determining Aedes distribution patterns and targeting areas for control (Sithiprasasna et al., 2004; Moreno-Sanchez et al., 2006; Tsuda et al., 2006; Lozano-Fuentes et al., 2008; Chang et al., 2009). More innovative techniques include predicting highrisk transmission zones (Carbajo et al., 2001; Ali et al., 2003; Getis et al., 2003; Chansang and Kittayapong, 2007). A number of advanced spatial and spatio-temporal analyses of DF cases in the Asia-Pacific region have also been undertaken (Hu et al., 2009, 2012; Li et al., 2012). However, there are few examples of applying spatial epidemiological tools to examine the dynamics of local sympatric Aedes populations and to develop targeted species-specific approaches to vector control in resource-limited settings.

This study characterises spatial patterns of Ae. aegypti and Ae. albopictus in San Jose village, Muntinlupa city in the Philippines. Specifically, the aim was to explore coexistence patterns and spatial clusters of Aedes populations and to predict vector density across the study area in order to identify high and low risk transmission zones at a fine spatial scale.

\section{Materials and methods}

\section{Study area and data collection}

Fortnightly, Aedes surveillance data (numbers of adult female Ae. aegypti and Ae. albopictus mosquitoes captured) were obtained from 50 double-sticky ovitraps (SOs) in San Jose village, Muntinlupa city for the period June-November 2011 (Fig. 1). San Jose village is a gated community of approximately 200 households located $30 \mathrm{~km}$ south of Manila (Duncombe and Marollano, 2011). The environment includes areas of dense bush land and has low population density $\left(2.48\right.$ per $\left.\mathrm{km}^{2}\right)$. Of the $50 \mathrm{SOs}, 48$ were placed under cover outside private homes and two were situated in public spaces near a community café. Households were selected using a spatial grid sampling design to facilitate geospatial analysis. Final selection of households was determined based on: (i) consent to participate by householders; (ii) accessibility of the property; and (iii) an adequate level of personal safety for fieldworkers.

SOs, designed to attract gravid adult female Aedes mosquitoes, are valuable tools for entomological surveillance because they are relatively inexpensive, portable and do not require electricity (Ritchie et al., 2004; Morrison et al., 2008; Chadee and Ritchie, 2010). They each comprise a top bucket lined with a polybutylene adhesive panel and a base bucket filled with water containing $0.5 \mathrm{~g}$ of organic attractant (i.e. lucerne) and a pellet of methoprene, which is an insect growth regulator that prevents mosquito maturation (Ritchie and Russell, 2002; Ritchie et al., 2003). These SOs were used throughout the work presented here with the base bucket cleaned and the water and pellets replenished each week.

During fieldwork, Aedes mosquitoes were extracted from the adhesive panel of each $\mathrm{SO}$, stored in individual plastic vials (pooled by $\mathrm{SO}$ ) and transported to the Medical Entomology Laboratory at the Research Institute of Tropical Medicine (RITM), Muntinlupa, 


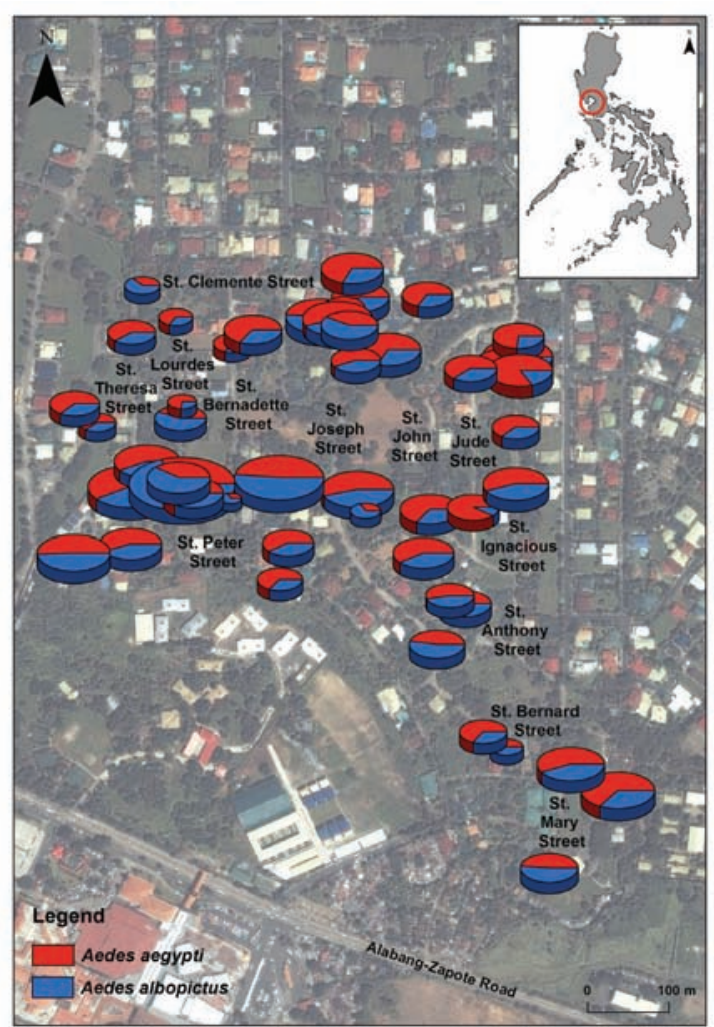

Fig. 1. Average fortnightly numbers of Ae. aegypti and Ae. albopictus for the study period (June-November 2011), sized according to average number of mosquitoes per sticky ovitrap (SO). See insert for the location of the study area in the Philippines.

the Philippines. Here, the species and sex of each mosquito was confirmed by Rueda's reference key via a Leica EZ4 stereo microscope (Rueda, 2004); approximately $5 \%$ of mosquitoes were damaged in or on removal from SOs and were visually identified in the field for species and sex.

One household (SO no. 18) was sent a letter (in English and Tagalog) in September following consistently high numbers of Aedes mosquitoes collected in this particular SO, and reports of suspected DF cases in the village. The letter informed the householders of the high numbers of Aedes found in the SO and suggested cleaning roof gutters and covering a nearby drain to remove possible breeding sites and reduce the likelihood of DF infection. The householders followed these recommendations and the number of Aedes collected in the SO decreased in the following weeks. Another household (SO no. 33) commenced indoor and outdoor insecticide spraying twice weekly in September 2011, subsequent to the hospitalization of multiple household members with suspected DF. Both of these incidents may have affected the number of adult female Aedes mosquitoes identified in SOs.

\section{Spatial cluster analysis}

Preliminary associations between fortnightly numbers of adult female Ae. aegypti and Ae. albopictus mosquitoes were visualised via mapping in the GIS software ArcMap, version 10.0 (ESRI; Redlands, USA).

The detection of spatial clustering within the study area during the study period was performed using the spatial scan statistic assuming a retrospective Poisson probability model in the SaTScan software, version 8.0 (Kulldorff, 1997). The spatial scan statistic centres a circular, isotropic window on each of the possible centroids throughout the geographical area, calculating the observed and expected number of mosquitoes in each circle and identifying the most likely and secondary (high or low) clusters. Markov chain Monte Carlo $($ MCMC) simulations $(\mathrm{n}=999)$ were conducted under the assumption of spatial randomness to determine the statistical significance of the identified clusters. The case data used for the spatial Poisson model was the sum of the mosquitoes for the study period by $\mathrm{SO}$ for a given species. The population was the total number of Aedes mosquitoes by SO for the study period. To avoid duplication of results (i.e. testing different sides of the same ratio) only one species was required for testing of cases; we selected Ae. aegypti. The maximum spatial cluster size was equal to $50 \%$ of the population at risk and the statistical significance of the clusters was specified at $\mathrm{P}=0.05$. Due to the relatively short study period, spatio-temporal analysis was not performed.

\section{Spatial interpolation}

Spatial autocorrelation was assessed by generating exponential semivariograms using the variog function in the $G e o R$ package of $\mathrm{R}$ statistical software ( $\mathrm{R}$ Development Core Team, 2012). Semiovariograms were created by species for each of the 10 time periods (fortnights), as well as for the total study period. Data comprised the total number of adult female Ae. aegypti and Ae. albopictus at each of the $50 \mathrm{SO}$ georeferenced locations for each time period. Covariance parameters were estimated by fitting weighted least squares (WLS) models to the semivariograms using the variofit function in the GeoR package.

Spatial interpolation methods are valuable for data where the variable of interest (e.g. mosquito density) is spatially continuous but is only measured at selected sites (e.g. in SOs). Kriging (Matheron, 1963) is a robust spatial interpolation technique that creates a 
prediction surface based on measured values (e.g. mosquito density in SOs), distance to prediction locations and a mathematical model of spatial autocorrelation informed by semivariogram covariance parameters (Pfeiffer et al., 2008). Ordinary kriging was selected as the most appropriate kriging model because it assumes an unknown mean which is defined by nearby data values; and thus, is more dynamic than other kriging models, such as simple kriging, which uses a pre-defined known mean for all locations.

For time periods in which spatial autocorrelation was detected using the semivariograms, a grid of 4,900 prediction locations $(70 \times 70 \mathrm{~m})$ covering the study area was created. Each grid location was rectangular because of the shape of the study site and measured 12 by $17 \mathrm{~m}$. Ordinary kriging was performed on the grid using the pois.krige and pois.glm.control functions in the GeoRglm package of R, and Aedes density estimates (counts) were obtained for each prediction location. The MCMC Metropolis-Hastings algorithm based on Langevin-Hastings updates and parameterisation specified in semivariography was employed to simulate the conditional distributions for gridded locations given data from SOs (Papaspiliopoulus et al., 2003). The number of MCMC iterations performed was 1,000, thinning was specified as 1 and burn-in set to 0 . The proposal variance was scaled to a value between 0.5 and 0.7 , assessed by trial and error for each time period, to ensure that approximately $60 \%$ of the proposals were accepted as required by Langevin-Hastings updates (Christensen and Waagepetersen, 2002). Further details of ordinary kriging using $\mathrm{R}$ are available elsewhere (Christensen and Ribeiro, 2012). The gridded kriging estimates were exported to ArcMap using raster image files created in the $\mathrm{R}$ rgdal package.

\section{Results}

The spatial distribution of Ae. aegypti and Ae. albopictus, and average number of mosquitoes at each SO location in the study area, is presented in Fig. 1. The temporal pattern of Aedes populations through the study period showed that Ae. aegypti mosquitoes are dominant in the first half of the study period only (Fig. 2). Ae. aegypti mosquito numbers increased from June to July and rapidly declined towards August. They increased again from August to September, though not as markedly and then declines towards November. Conversely, Ae. albopictus numbers declined from June to July, increased to a peak in September and decreased again towards November.

Spatio-temporal population dynamics are explored for Aedes mosquitoes in Figs. 3 and 4. As expected, $A e$. aegypti specimens were more common in the residential areas in the northeast, while Ae. albopictus was dominant in the southwest where there is more vegetation and fewer houses. The maps show opposing areas of high vector density suggesting that, despite Ae. aegypti and Ae. albopictus residing sympatrically in San Jose village, only one species dominates each geographical area. These patterns were confirmed by spatial cluster detection, with a significantly low primary cluster of Ae. aegypti in the west (relative risk $(\mathrm{RR})=0.60, \mathrm{P}=0.002)$ and a significantly high, larger secondary cluster of Ae. aegypti in the east $(\mathrm{RR}=1.25, \mathrm{P}=0.002)($ Fig. 5$)$.

Spatial autocorrelation was apparent in Ae. aegypti populations for the following periods: June 14November 2 (whole study period); June 14-28; July 26-August 9; and October 4-18 (Fig. 6). Spatial autocorrelation was evident for Ae. albopictus mosquitoes for the period October 4-18 only. Semivariograms
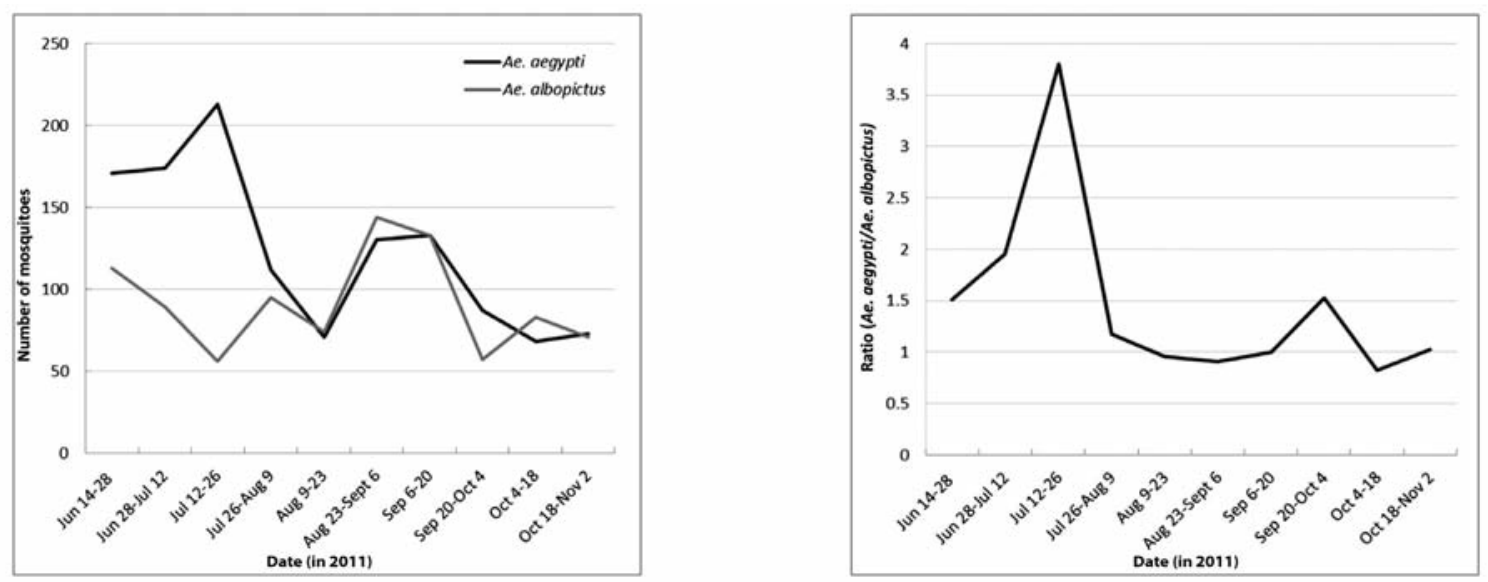

Fig. 2. The total number and ratio of Ae. aegypti and Ae. Albopictus mosquitoes by fortnight for the study period. 

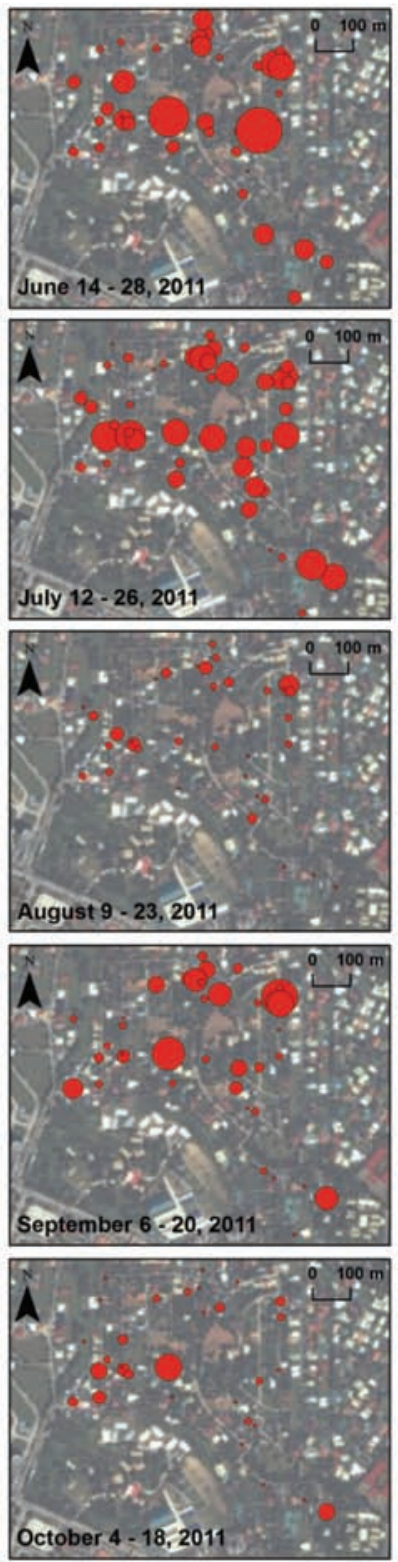

Fig. 3. Total fortnightly numbers of Ae. aegypti by time period, sized according to the total number of mosquitoes per sticky ovitrap (OS).

indicate spatial autocorrelation up to a distance of 100 $\mathrm{m}$; that is, the number of Aedes mosquitoes at a given SO location was autocorrelated with the number of mosquitoes at other SOs located within $100 \mathrm{~m}$.

Fig. 7 presents kriging maps of interpolated densities for Ae. aegypti and Ae. albopictus in the study site for the given time periods. Kriging estimates for Ae. aegypti populations reveal similar patterns through time periods with high density in the eastnortheast of the study area, except for October 4-18 where density was highest in the west. For the same period, kriging estimates for Ae. albopictus demonstrated high density in the south of the study area.
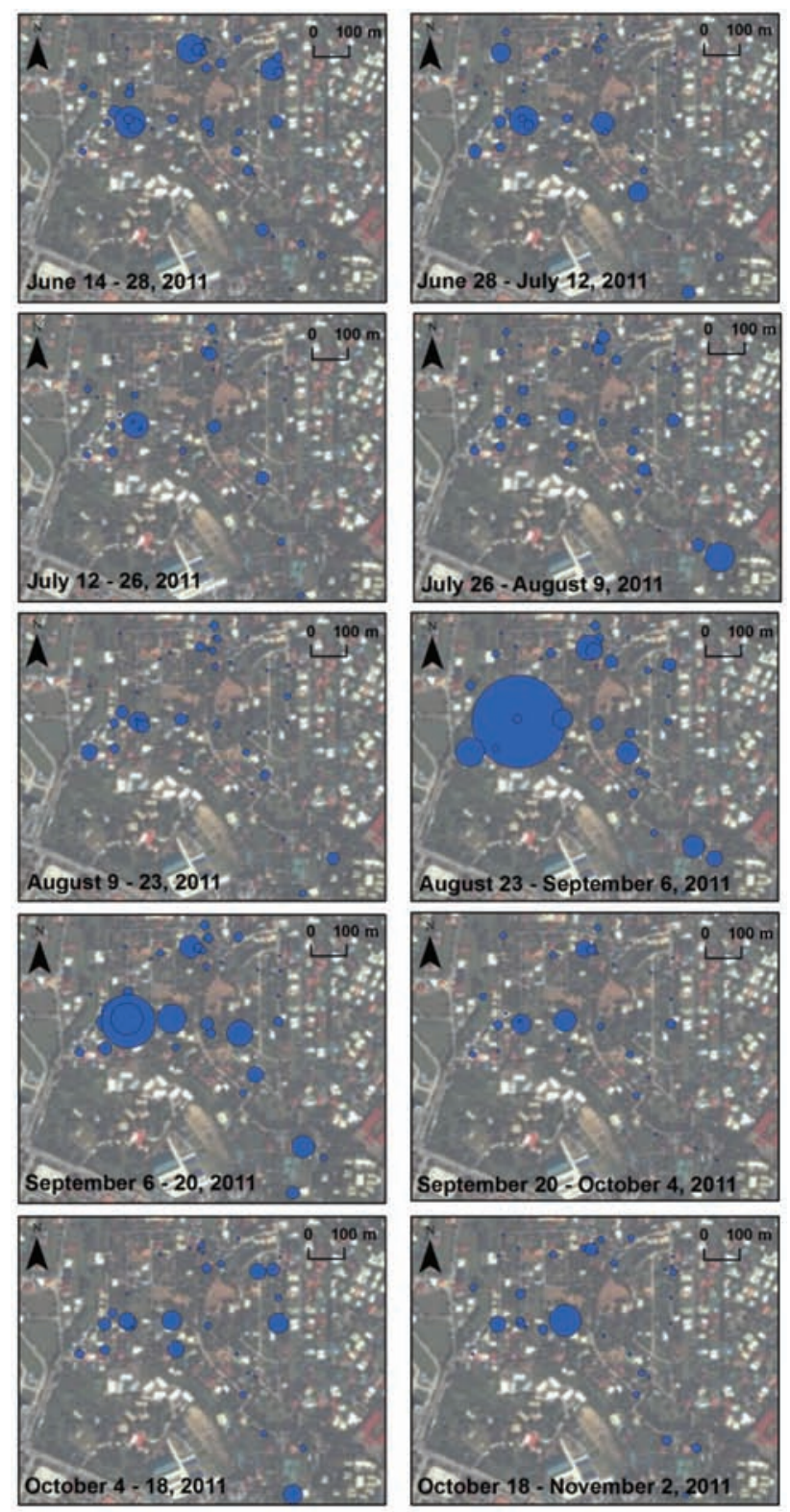

Fig. 4. Total fortnightly numbers of Ae. albopictus by time period, sized according to the total number of mosquitoes per sticky ovitrap (OS).

\section{Discussion}

Our results differ from findings from a recent multisite study undertaken in Southeast Asian countries, where Ae. albopictus was not identified in the Philippines or neighbouring countries (Arunachalam et al., 2010). Additionally, a larval and pupal survey undertaken in Muntinlupa city in 2008 identified Ae. aegypti but not Ae. albopictus mosquitoes (Cruz et al., 2008). We demonstrate here coexistence of Ae. aegypti and Ae. albopictus in San Jose village in the Philippines; which is consistent with a 25 -year-old study undertaken in cemeteries in Manila, finding the 


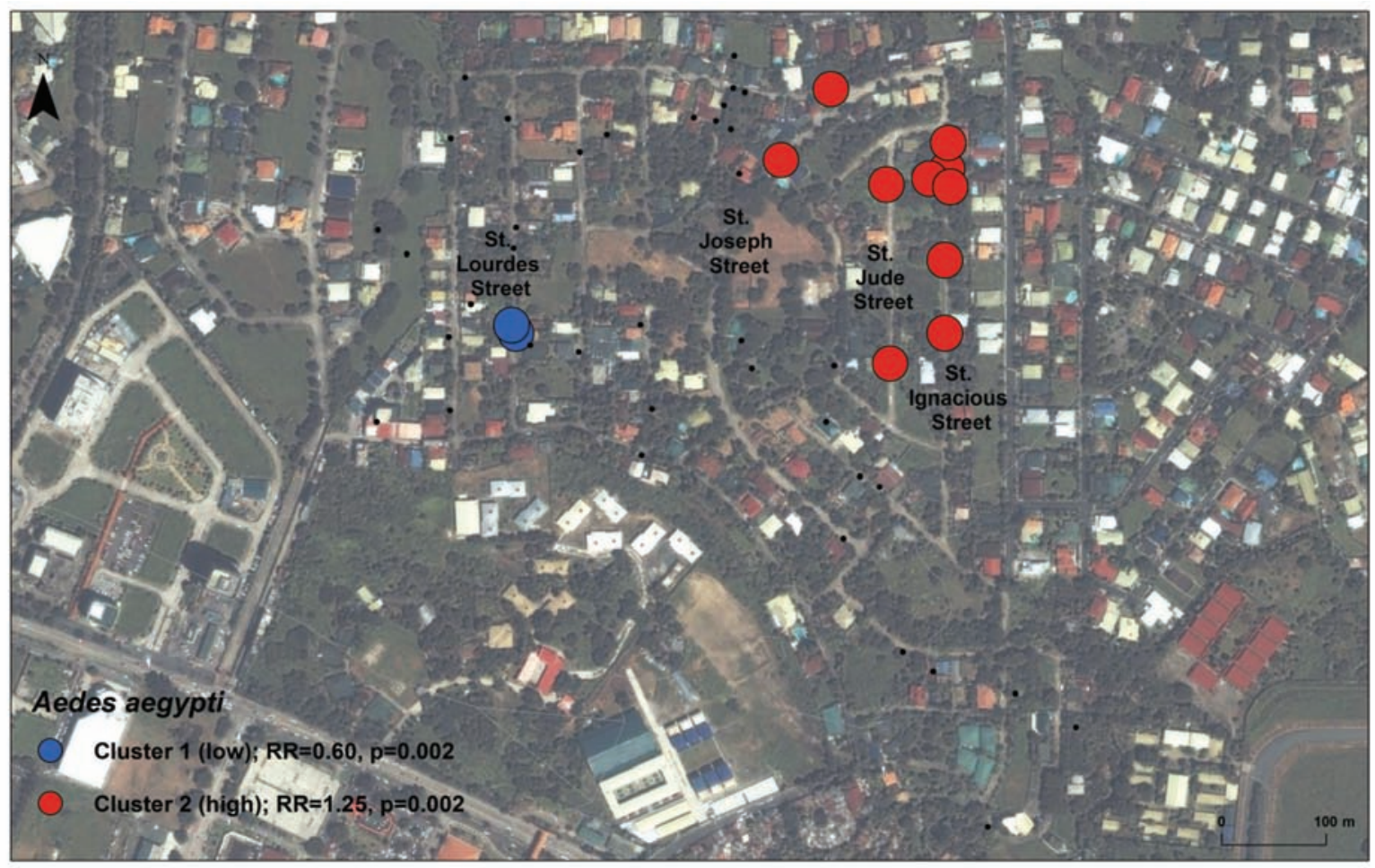

Fig. 5. Spatial cluster detection for total numbers of Ae. aegypti and Ae. albopictus mosquitoes for the study period.

presence of both species and showing that Ae. albopictus dominate vegetated areas (Schultz, 1989, 1993). More recent entomological surveillance, undertaken in a semi-rural area of the Philippine island of Palawan, reveals comparable rates of $A e$. aegypti and Ae. albopictus, confirming our findings (unpublished data). A number of other countries in Asia, including Singapore, Thailand, Malaysia, and Vietnam, have also reported Aedes coexistence (Chan et al., 1971; Yap, 1975; Yap and Thiruvengadam, 1979; Kay et al., 2002).

To our knowledge, this is the first study to use robust geostatistical methods to explore sympatric Ae. aegypti and Ae. albopictus populations. We found that, although Aedes mosquitoes reside sympatrically, they prefer different locations in the study area. Generally, where density of Ae. aegypti was found to be high, that of Ae. albopictus was low, and vice versa. This is likely due to preferences for different environments (residential versus rural) and interspecies competition resulting in one species dominating a given geographical area, as shown by Juliano et al. (2004) and Tsuda et al. (2006). Indeed, increased numbers of Ae. albopictus mosquitoes in vegetative areas later in the wet season may extend spatial and temporal opportunities for DF transmission, which would not be possible if Ae aegypti were the sole vector. Exploration of sympatric Aedes populations is impor- tant because evidence suggests that greater competition resulting from coexistence of species may result in increases in vector competence (Alto et al., 2008). Physiological characteristics of adult mosquitoes, including body size and wing length may be influenced by competition and other ecological interactions in the larval stage and may, therefore, affect adult vector competence for pathogens such as DF. However, most of the evidence for interspecies competition comes from laboratory-based studies that do not provide information intended for the control of diseases such as DF in real-world settings.

There was no observed spatial autocorrelation in the majority of time periods throughout the study, possibly due to small numbers in the dataset for those time periods. Spatial autocorrelation for Ae. aegypti in June 14-November 2, June 14-28, July 26-August 9 and October 4-18, and for Ae. albopictus during October 4-18, was found up to a distance of 0.001 decimal degrees (approximately $100 \mathrm{~m}$ ). This information is potentially useful for vector surveillance because it indicates that SOs could be placed approximately 100 $\mathrm{m}$ apart to provide comprehensive surveillance coverage, i.e. closer placement would waste surveillance resources.

Interpolated vector density maps showed similar high vector density zones throughout the study period for Ae. aegypti populations in the east of the study 

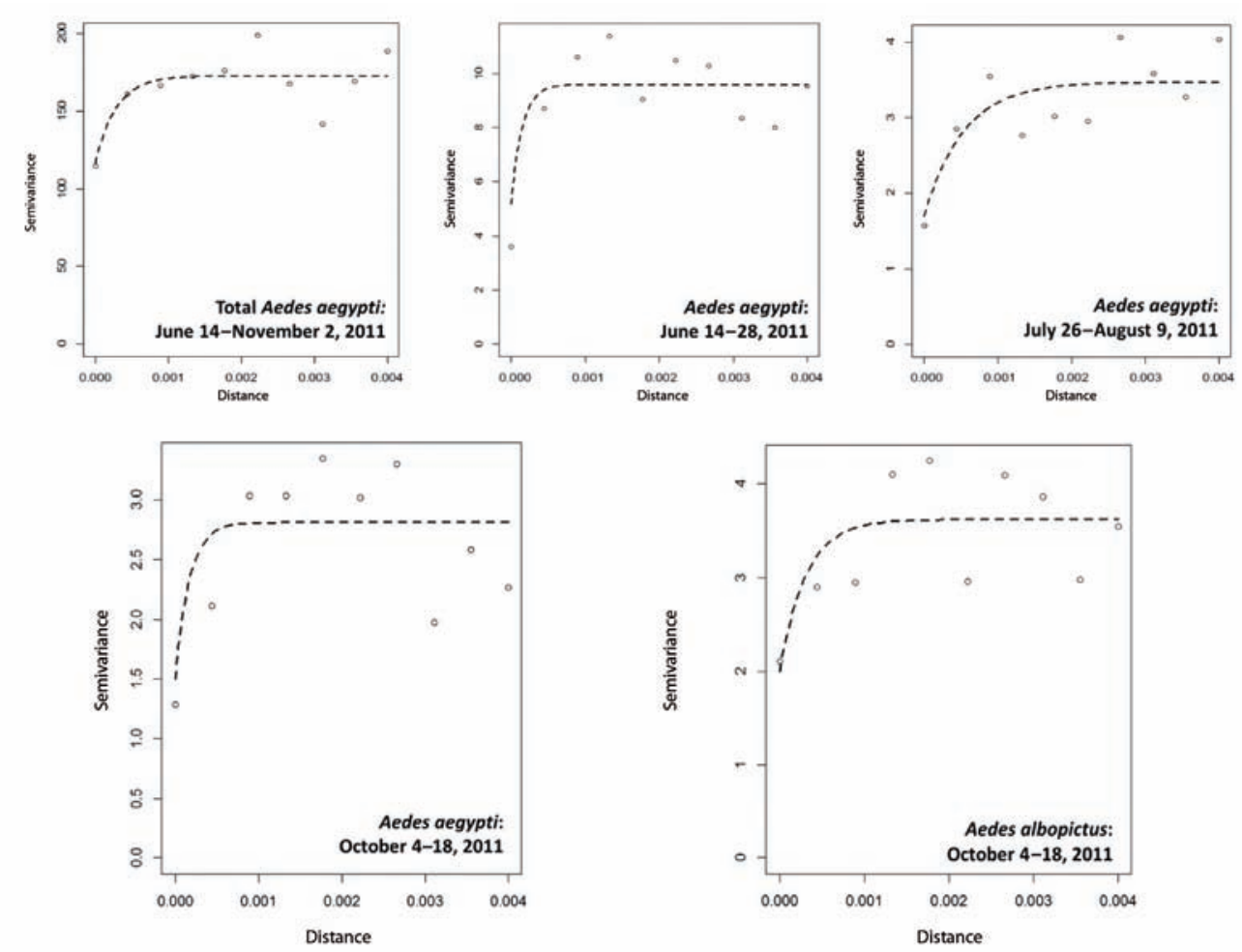

Fig. 6. Semivariograms demonstrating spatial autocorrelation, by time period and species, up to a lag distance of 0.001 decimal degrees (equivalent to approximately $100 \mathrm{~m}$ at the Equator).

area. However, high vector density for Ae. aegypti during October 4-18 was seen in the west and Ae. albopictus density was highest in the south of the study area. Spatial differences in species preferred locations may be influenced by habitat characteristics previously mentioned (e.g. residential versus vegetative location), host location and ovipositon behaviour. Indeed, Ae. aegypti are more anthropophilic than Ae. albopictus, which might also blood feed on vertebrates other than humans, and thus are more likely to oviposit inside homes or in close proximity to humans (Barrera, 1996). Mosquito density maps showing high transmission zones can be used to advocate for better resources and improved targeting of prevention activities. Evidence supports prevention and control programmes at the household level or container level for maximum protection (Arunachalam et al., 2010). Maps may be used to identify areas of low transmission risk so that valuable resources may be oriented away from these areas and better utilised in other, high-risk, areas.

The development of integrated, sub-national surveillance systems, such as spatial decision support systems (SDSS), could assist the planning, targeting and implementation of prevention and control programmes (Kelly et al., 2010, 2011, 2012; Eisen and Eisen, 2011). In particular, SDSS could improve the speed, accuracy and efficiency of control activities during an epidemic, such as IRS, where maintaining a high spraying coverage rate is essential for ensuring control effectiveness and limiting DF transmission (VazquezProkopec et al., 2010). SDSS may also determine risk areas for DHF and DSS epidemics following the introduction of a new virus serotype in a population with herd immunity to previous virus serotypes. Additionally, SDSS may improve the coordination of control activities between localities, facilitate resource allocation decisions via user-defined reports and enable data collection and reporting standardisation across large countries like the Philippines.

The assessment of some vector control strategies has been undertaken in the Philippines, including the use of the microbial agent Bacillus thuringiensis israelensis in water containers (de Melo-Santos et al., 2001; Mahilum et al., 2005), and insecticide-treated curtains inside houses (Madarieta et al., 1999). A multifaceted vector control approach, that includes environmental management and chemical and biological methods, has also been suggested ( $\mathrm{Su}, 2008)$. In 2011, the Department of Science and Technology (DOST) commenced a multimillion-peso project to distribute ovicidal/larvicidal (OL) traps to schools and households nationwide with the aim of reducing the number of female Aedes mosquitoes and therefore interrupting 

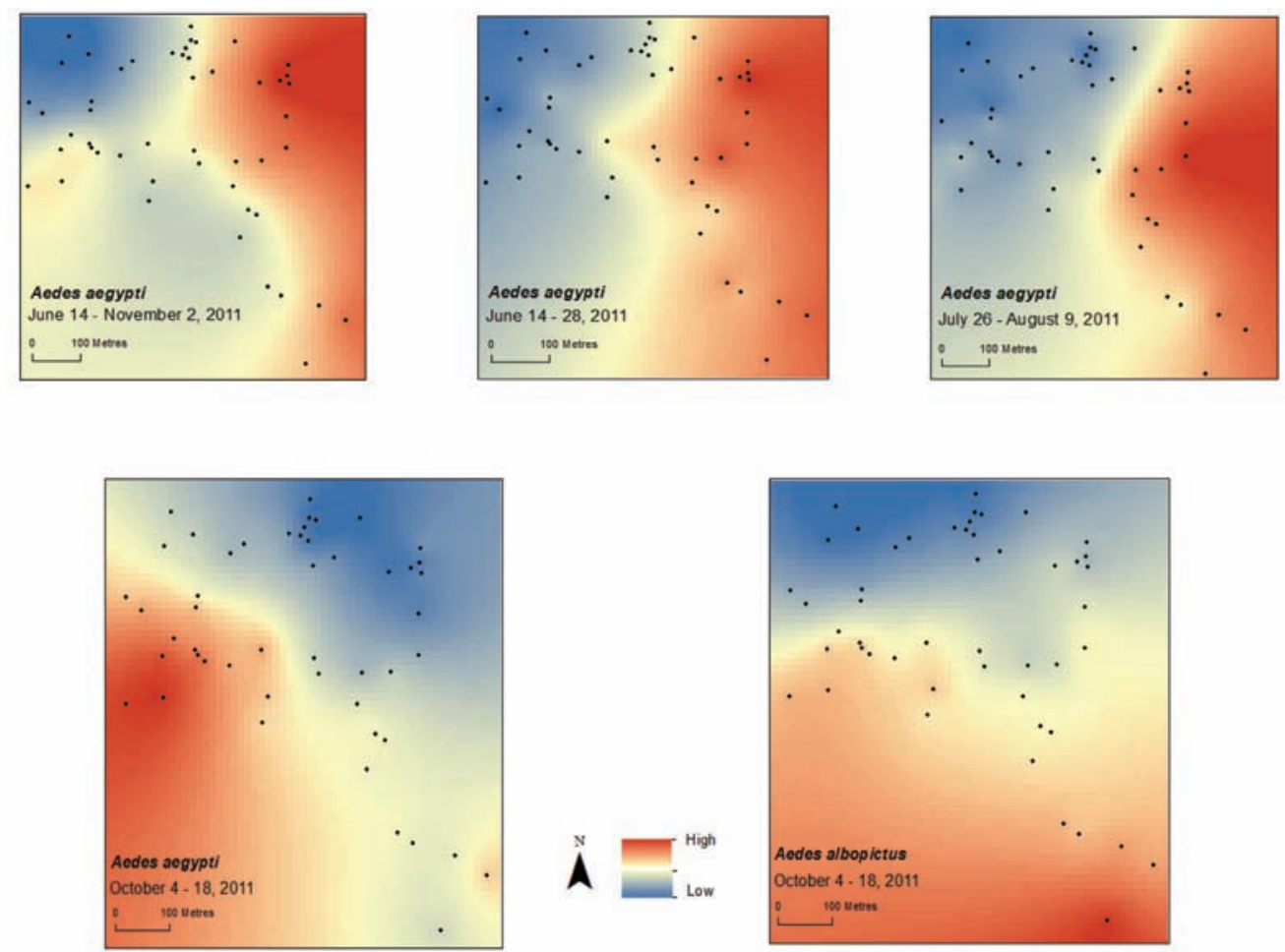

Fig. 7. Ordinary kriging estimates by species for each time point demonstrating spatial autocorrelation for June 14-28, July 26August 9 and October 4-18, as well as for the whole study period (June 14-November 2).

DF transmission. Unfortunately, deficient field testing of OL traps prior to commencement and a lack of community education on the use and maintenance of the traps (potentially transforming them into Aedes breeding sites) may limit the effectiveness of the project (Briones et al., 2012). Other concerns include ongoing maintenance costs of OL traps for schools and households, and the availability of local health staff to assist the placement of OL traps, therefore, reducing their capacity to perform other duties.

Neighbouring countries have also tried a number of vector control strategies for DF prevention. In Cambodia, for example, larvicidal and insecticidal controls have been tested, including the use of larvivorous fish (Poecilia reticulate) in rural communities to control Ae. aegypti in water storage containers (Seng et al., 2006, 2008a, 2008b, 2008c; Suaya et al., 2007). In Vietnam, the crustacean Mesocyclops (Copepoda) has been utilised in a preventative vector control programme based on community-mobilization (Kay et al., 2002; Kay and Vu, 2005). The bacterium Wolbachiaused to shorten the lifespan of Ae. aegypti mosquitoes - is currently being tested in Vietnam (Jeffery et al., 2009; Hurst et al., 2012). A more comprehensive, but resource-intensive, vector control programme was implemented in the Plaeng Yao district, Thailand, employing community education, physical removal of oviposition sites, lethal ovitraps, biological controls and insecticides (Kittayapong et al., 2006). Further research in this area should focus on establishing sustainable vector control programmes employing community mobilization and considering local ecological, biological and social factors (Heintze et al., 2007; Arunachalam et al., 2010; van den Berg et al., 2012). Additionally, the response of Ae. albopictus to vector control strategies should be investigated following difficulties implementing control programmes in other parts of the world (Paupy et al., 2009).

The current study has some limitations, including the short study period (5 months). A longer time series is required to establish seasonal patterns of Aedes mosquitoes and determine the feasibility of using SOs in areas with different socio-demographic and environmental characteristics. Additionally, the spatial sampling frame was compromised because of concerns with property accessibility and personal safety. Another limitation was the lack of funding available for virologic surveillance, such as reverse transcription-polymerase chain reaction (RT-PCR) assays, which can be undertaken on field-collected Aedes mosquitoes to determine virus infection rates and serotypes by species, and may act as an early warning system for epidemics (Chow et al., 1998). However, mosquitoes have been stored appropriately for use if 
funding becomes available. Although spatial analysis was performed based on a latitude and longitude geographical coordinate system, which may have impacted distance measures provided, the study area was close to the Equator, and thus, the results were likely unaffected.

Our results show that Aedes mosquitoes cohabitate in San Jose village but have opposing spatial clustering patterns, suggesting interspecies competition and differing habitat preferences. Mosquito density maps identify potential high and low risk DF transmission zones for directing prevention and control activities, and may be used to advocate the development of targeted local-level surveillance such as SDSS for integrated DF management in the Philippines. We recommend further studies to confirm our findings and determine DF infection rates and seroprevalence rates in Aedes field-collected mosquitoes to provide evidence for the association between vector density and DF transmission. Applied research identifying, implementing and evaluating sustainable community-based vector control programmes should also be prioritised. Additionally, confirmation of the vector competence of local Ae. albopictus populations is required to determine whether ongoing habitat expansion in locations with sympatric Aedes populations would increase the global health threat from DF.

\section{Ethical approvals}

Human research ethics applications submitted to the RITM Institutional Review Board (2011-10) and the University of Queensland Medical Research Ethics Committee (2011000490) were approved.

\section{Acknowledgements}

We sincerely thank the San Jose village householders for permitting us access to their properties each week and for their hospitality and kindness. Special thanks to Caesar, Jonathan, Jesse and Neil for their commitment during the fieldwork. We also thank Ava Kristy Sy and Inez Medado (Molecular Biology Department, RITM); Christine Joy Dureza (Epidemiology and Biostatistics Department, RITM); the Medical Entomology and Parasitology Departments (RITM); and the Muntinlupa city Health Office for their advice and support during this study. Thanks to the Queensland Tropical Public Health Unit for donating the SOs and providing valuable advice. Fieldwork (JD) was supported by an Australian Endeavour Research Fellowship (\#2234_2011). ACAC is supported by an Australian National Health and Medical Research Council Career Development Award (\#631619).

\section{References}

Ali M, Wagatsuma Y, Emch M, Breiman RF, 2003. Use of a geographic information system for defining spatial risk for dengue transmission in Bangladesh: role for Aedes albopictus in an urban outbreak. Am J Trop Med Hyg 69, 634-640.

Alto BW, Lounibos LP, Mores CN, Reiskind MH, 2008. Larval competition alters susceptibility of adult Aedes mosquitoes to dengue infection. Proc Biol Sci 275, 463-471.

Arunachalam N, Tana S, Espino F, Kittayapong P, Abeyewickreme W, Wai KT, Tyagi BK, Kroeger A, Sommerfeld J, Petzold M, 2010. Eco-bio-social determinants of dengue vector breeding: a multicountry study in urban and periurban Asia. Bull World Health Organ 88, 173-184.

Barrera R, 1996. Competition and resistance to starvation in larvae of container-inhabiting Aedes mosquitoes. Ecol Entomol 21, 117-127.

Bossert TJ, Beauvais JC, 2002. Decentralization of health systems in Ghana, Zambia, Uganda and the Philippines: a comparative analysis of decision space. Health Policy Plann 7, 1431.

Briones AV, Garbo AG, Casa EP, Bion HH, Almanzor NE, Bernardo ST, 2012. Effects of aqueous and pelletised admixture of Piper nigrum L. on the oviposition behavior of Aedes aegypti mosquitoes and its larvicidal activity. Acta Med Philipp 46, 55-58.

Carbajo AE, Schweigmann N, Curto SI, de Garin A, Bejaran R, 2001. Dengue transmission risk maps of Argentina. Trop Med Int Health 6, 170-183.

Chadee D, Ritchie SA, 2010. Efficacy of sticky and standard ovitraps for Aedes aegypti in Trinidad, West Indies. J Vect Ecol 35, 395-400.

Chan YC, Ho BC, Chan KL, 1971. Aedes aegypti (L.) and Aedes albopictus (Skuse) in Singapore City. 5. Observations in relation to dengue haemorrhagic fever. Bull World Health Organ 44, 651-657.

Chang AY, Parrales ME, Jimenez J, Sobieszczyk ME, Hammer SM, Copenhaver DJ, Kulkarni RP, 2009. Combining Google Earth and GIS mapping technologies in a dengue surveillance system for developing countries. Int J Health Geogr 8, 49-60. Chansang C, Kittayapong P, 2007. Application of mosquito sampling count and geospatial methods to improve dengue vector surveillance. Am J Trop Med Hyg 77, 897-902.

Chow VT, Chan YC, Yong R, Lee KM, Lim LK, Chung YK, Lam-Phua SG, Tan BT, 1998. Monitoring of dengue viruses in field-caught Aedes aegypti and Aedes albopictus mosquitoes by a type-specific polymerase chain reaction and cycle sequencing. Am J Trop Med Hyg 58, 578-586.

Christensen OF, Ribeiro PJ, 2012. geoRglm - a package for generalised linear spatial models. Available at: http://cran.opensourcesolution.org/web/packages/geoRglm/geoRglm.pdf (accessed on September 2012). 
Christensen OF, Waagepetersen R, 2002. Bayesian prediction of spatial count data using generalised linear mixed models. Biometrics 58, 280-286.

Cruz EI, Salazar FV, Porras E, Mercado R, Orais V, Bunyi J, 2008. Entomological survey of dengue vectors as basis for developing vector control measures in Barangay Poblacion, Muntinlupa city, Philippines, 2008. Dengue Bull 32, 167-170.

de Melo-Santos MA, Sanches EG, de Jesus FJ, Regis L, 2001. Evaluation of a new tablet formulation based on Bacillus thuringiensis sorovar. israelensis for larvicidal control of Aedes aegypti. Mem Inst Oswaldo Cruz 96, 859-860.

Duncombe J, Clements A, Hu W, Weinstein P, Ritchie SA, Espino FE, 2012. Geographical information systems for dengue surveillance. Am J Trop Med Hyg 86, 753-755.

Duncombe J, Marollano K, 2011. Dengue vector surveillance methods in Muntinlupa city, Philippines. Western Pac Surveill Response J 2.

Eisen L, Eisen RJ, 2011. Using geographic information systems and decision support systems for the prediction, prevention, and control of vector-borne diseases. Annu Rev Entomol 56, 41-61.

Eisen L, Lozano-Fuentes S, 2009. Use of mapping and spatial and space-time modeling approaches in operational control of Aedes aegypti and dengue. PLoS Negl Trop Dis 3, e411.

Getis A, Morrison AC, Gray K, Scott TW, 2003. Characteristics of the spatial pattern of the dengue vector, Aedes aegypti, in Iquitos, Peru. Am J Trop Med Hyg, 69, 495-505.

Gratz NG, 2004. Critical review of the vector status of Aedes albopictus. Med Vet Entomol, 18, 215-227.

Gubler DJ, Kuno G, 1997. Dengue and dengue hemorrhagic fever, Wallingford, England, New York, CAB International.

Heintze C, Velasco GM, Kroeger A, 2007. What do community-based dengue control programmes achieve? A systematic review of published evaluations. Trans R Soc Trop Med Hyg 101, 317-325.

Higa Y, 2011. Dengue vectors and their spatial distribution. Trop Med Health 39, 17-27.

Hu W, Clements A, Williams G, Tong S, 2009. Dengue fever and El Nino-Southern Oscillation in Queensland, Australia: a time series predictive model. Occup Environ Med 67, 307-311.

Hu W, Clements A, Williams G, Tong S, Mengersen K, 2012. Spatial patterns and socioecological drivers of dengue fever transmission in Queensland, Australia. Environ Health Persp 120, 260-266.

Hurst TP, Pittman G, O’Neill SL, Ryan PA, Nguyen HL, Kay $\mathrm{BH}, 2012$. Impacts of Wolbachia infection on predator prey relationships: evaluating survival and horizontal transfer between wMelPop infected Aedes aegypti and its predators. J Med Entomol 49, 624-630.

Jeffery JA, Thi YN, Nam VS, Nghia T, Hoffmann AA, Kay BH, Ryan PA, 2009. Characterizing the Aedes aegypti population in a Vietnamese village in preparation for a Wolbachia-based mosquito control strategy to eliminate dengue. PLoS Negl
Trop Dis 3, e552.

Juliano S, Lounibos LP, O’Meara G, 2004. A field test for competitive effects of Aedes albopictus on A. aegypti in South Florida: differences between sites of coexistence and exclusion? Oecologia 139, 583-593.

Kay BH, Nam VS, Tien TV, Yen NT, Phong TV, Diep VT, Ninh TU, Bektas A, Aaskov JA, 2002. Control of Aedes vectors of dengue in three provinces of Vietnam by use of Mesocyclops (Copepoda) and community-based methods validated by entomologic, clinical, and serological surveillance. Am J Trop Med Hyg 66, 40-48.

Kay BH, Vu SN, 2005. New strategy against Aedes aegypti in Vietnam. Lancet 365, 613-617.

Kelly GC, Hii J, Batarii W, Donald W, Hale E, Nausien J, Pontifex S, Vallely A, Tanner M, Clements A, 2010. Modern geographical reconnaissance of target populations in malaria elimination zones. Malar J 9, 289-301.

Kelly GC, Seng CM, Donald W, Taleo G, Nausien J, Batarii W, Iata H, Tanner M, Vestergaard LS, Clements ACA, 2011. A spatial decision support system for guiding focal indoor residual interventions in a malaria elimination zone. Geospat Health 6, 21-31.

Kelly GC, Tanner M, Vallely A, Clements A, 2012. Malaria elimination: moving forward with spatial decision support systems. Trends Parasitol 28, 297-304.

Kittayapong P, Chansang U, Chansang C, Bhumiratana A, 2006. Community participation and appropriate technologies for dengue vector control at transmission foci in Thailand. $\mathrm{J}$ Am Mosq Control Assoc 22, 538-546.

Kulldorff M, 1997. A spatial scan statistic. Commun Stat Theory Methods 26, 1481-1496.

Lambrechts L, Scott TW, Gubler DJ, 2010. Consequences of the expanding global distribution of Aedes albopictus for Dengue virus transmission. PLoS Negl Trop Dis 4, e646.

Li Z, Yin W, Clements A, Williams G, Lai S, Zhou H, Zhao D, Guo Y, Zhang Y, Wang J, Hu W, Yang W, 2012. Spatiotemporal analysis of indigenous and imported dengue fever cases in Guangdong province, China. BMC Infect Dis, 12, 132-141.

Lounibos LP, 2002. Invasions by insect vectors of human disease. Annu Rev Entomol 47, 233-266.

Lozano-Fuentes S, Elizondo-Quiroga D, Farfan-Ale JA, LoronoPino MA, Garcia-Rejon J, Gomez-Carro S, Lira-Zumbardo V, Najera-Vazquez R, Fernandez-Salas I, Calderon-Martinez J, Dominguez-Galera M, Mis-Avila P, Morris N, Coleman M, Moore CG, Beaty BJ, Eisen L, 2008. Use of Google Earth to strengthen public health capacity and facilitate management of vector-borne diseases in resource-poor environments. Bull World Health Organ 86, 718-725.

Madarieta SK, Salarda A, Benabaye MRS, Bacus MB, Tagle JR, 1999. Use of permethrin-treated curtains for control of Aedes aegypti, in the Philippines. Dengue Bull 23, 51-54. 
Mahilum MM, Ludwig M, Madon MB, Becker N, 2005. Evaluation of the present dengue situation and control strategies against Aedes aegypti in Cebu city, Philippines. J Vector Ecol 30, 277-283.

Matheron, G, 1963. Principles of geostatistics. Econ Geol 58, 1246-1266.

Moreno-Sanchez R, Hayden M, Janes C, Anderson G, 2006. A web-based multimedia spatial information system to document Aedes aegypti breeding sites and dengue fever risk along the US-Mexico border. Health Place 12, 715-727.

Morrison AC, Zielinski-Gutierrez E, Scott TW, Rosenberg R, 2008. Defining challenges and proposing solutions for control of the virus vector Aedes aegypti. PLoS Med 5, e68.

Ooi EE, Goh KT, Gubler DJ, 2006. Dengue prevention and 35 years of vector control in Singapore. Emerg Infect Dis 12, 887 893.

Papaspiliopoulus O, Roberts GO, Skold M, 2003. Non-centered parameterizations for hierarchical models and data augmentation, Oxford: Oxford University Press.

Paupy C, Delatte H, Bagny L, Corbel V, Fontenille D, 2009. Aedes albopictus, an arbovirus vector: from the darkness to the light. Microbes Infect 11, 1177-1185.

Pfeiffer DU, Robinson TP, Stevenson M, Stevens KB, Rogers DJ, Clements AC, 2008. Spatial analysis in epidemiology. Oxford: Oxford University Press.

Powers AM, Logue CH, 2007. Changing patterns of chikungunya virus: re-emergence of a zoonotic arbovirus. J Gen Virol 88, 2363-2377.

R Development Core Team, 2012. R: a language and environment for statistical computing. Vienna: R Foundation for Statistical Computing.

Ritchie SA, Long S, Hart A, Webb CE, Russell RC, 2003. An adulticidal sticky ovitrap for sampling container-breeding mosquitoes. J Am Mosq Control Assoc 19, 235-242.

Ritchie SA, Long S, Smith G, Pyke A, Knox TB, 2004. Entomological investigations in a focus of dengue transmission in Cairns, Queensland, Australia, by using the sticky ovitraps. J Med Entomol 41, 1-4.

Ritchie SA, Russell RC, 2002. A review of the New Zealand mosquito surveillance programme. Available at: http://www.moh.govt.nz/notebook/nbbooks.nsf/0/39D9C87 B4168F39CCC256C37000B2732 (accessed on August 2012).

Rueda LM, 2004. Pictorial keys for the identification of mosquitoes (Diptera: Culicidae) associated with dengue virus transmission. Auckland: Magnolia Press.

Schultz GW, 1989. Cemetery vase breeding of dengue vectors in Manila, Republic of the Philippines. J Am Mosq Control Assoc 5, 508-513.

Schultz GW, 1993. Seasonal abundance of dengue vectors in Manila, Republic of the Philippines. Southern Asian J Trop Med Public Health 24, 369-375.

Seng CM, Setha T, Chanta N, Socheat D, Guillet P, Nathan MB,
2006. Inhibition of adult emergence of Aedes aegypti in simulated domestic water-storage containers by using a controlledrelease formulation of pyriproxyfen. J Am Mosq Control Assoc 22, 152-154.

Seng CM, Setha T, Nealon J, Chantha N, Socheat D, Nathan MB, 2008a. The effect of long-lasting insecticidal water container covers on field populations of Aedes aegypti (L.) mosquitoes in Cambodia. J Vector Ecol 33, 333-341.

Seng CM, Setha T, Nealon J, Socheat D, Chantha N, Nathan $\mathrm{MB}, 2008 \mathrm{~b}$. Community-based use of the larvivorous fish Poecilia reticulata to control the dengue vector Aedes aegypti in domestic water storage containers in rural Cambodia. J Vector Ecol 33, 139-144.

Seng CM, Setha T, Nealon J, Socheat D, Nathan MB, 2008c. Six months of Aedes aegypti control with a novel controlledrelease formulation of pyriproxyfen in domestic water storage containers in Cambodia. Southern Asian J Trop Med Public Health 39, 822-826.

Sithiprasasna R, Patpoparn S, Attatippaholkun W, Suvannadabba S, Srisuphanunt M, 2004. The geographic information system as an epidemiological tool in the surveillance of dengue virus-infected Aedes mosquitos. Southern Asian J Trop Med Public Health 35, 918-926.

Su GL, 2008. Correlation of climatic factors and dengue incidence in Metro Manila, Philippines. Ambio 37, 292-294.

Suaya JA, Shepard DS, Chang MS, Caram M, Hoyer S, Socheat D, Chantha N, Nathan MB, 2007. Cost-effectiveness of annual targeted larviciding campaigns in Cambodia against the dengue vector Aedes aegypti. Trop Med Int Health 12, 1026-1036.

Tsuda Y, Suwonkerd W, Chawprom S, Prajakwong S, Takagi M, 2006. Different spatial distribution of Aedes aegypti and Aedes albopictus along an urban-rural gradient and the relating environmental factors examined in three villages in northern Thailand. J Am Mosq Control Assoc 22, 222-228.

van den Berg $\mathrm{H}$, Velayudhan R, Ebol A, Catbagan BH jr, Turingan R, Tuso M, Hii J, 2012. Operational efficiency and sustainability of vector control of malaria and dengue: descriptive case studies from the Philippines. Malar J 11, 269.

Vazquez-Prokopec GM, Kitron U, Montgomery B, Horne P, Ritchie SA, 2010. Quantifying the spatial dimension of dengue virus epidemic spread within a tropical urban environment. PLoS Negl Trop Dis 4, e920.

WHO, 2012. Dengue and severe dengue: factsheet no. 117. Available at: http://www.who.int/mediacentre/factsheets/fs117/en/ (accessed on October 2012).

Yap HH, 1975. Distribution of Aedes aegypti (Linnaeus) and Aedes albopictus (Skuse) in small towns and villages of Penang Island, Malaysia - an ovitrap survey. Southern Asian J Trop Med Public Health 6, 519-524.

Yap HH, Thiruvengadam V, 1979. Relative abundance of Aedes aegypti (Linnaeus) and Aedes albopictus (Skuse) in different habitats. Med J Malaysia 34, 76-79. 